\title{
Preliminary computational experience with a descent level method for convex nondifferentiable optimization
}

\author{
U. Brännlund
}

Royal Institute of Technology, Stockholm, Sweden.

e-mail: uffe@math.kth.se

K.C. Kiwiel

System Research Institute, Warsaw, Poland.

e-mail: kiwielQibspan.waw.pl

\section{P.O. Lindberg}

Royal Institute of Technology, Stockholm, Sweden.

e-mail: pol@math.kth.se

\begin{abstract}
We report on numerical tests with our recently introduced descent level bundle method for convex minimization. The test problems include standard nonsmooth problems, eigenvalue problems, and Lagrangian duals of traveling salesman, capacitated lotsizing, hierarchical production planning and unit commitment problems.
\end{abstract}

\section{Keywords}

Nondifferentiable optimization, proximal bundle methods, level methods.

\section{INTRODUCTION}

We have recently introduced a descent bundle method Brännlund et al. (1995) for minimizing a (possibly nondifferentiable) convex function $f: \mathbb{R}^{N} \rightarrow \mathbb{R}$ over a nonempty closed convex set $S \subset \mathbb{R}^{N}$. We assume that at each $x \in S$ we can compute $f(x)$ and an arbitrary subgradient $g(x) \in \partial f(x)$.

At the $k$ th iteration, having generated linearizations $f^{j}(\cdot)=f\left(y^{j}\right)+\left\langle g\left(y^{j}\right), \cdot-y^{j}\right\rangle$ of $f$ at trial points $y^{j} \in S$ for $j=1: k$, we approximate $f$ from below by the piecewise linear 
cutting-plane model $\breve{f}^{k}=\max _{j \in J^{k}} f^{j}$, where $J^{k} \subset\{1: k\}$ contains $k$ and at most $N$ other indices. We set

$y^{k+1}=\arg \min \left\{\left|x-x^{k}\right|^{2} / 2: x \in S, \check{f}^{k}(x) \leq f_{\text {lev }}^{k}\right\}$,

where the prox-center $x^{k}$ usually has the best $f$-value among the points $\left\{y^{j}\right\}_{j=1}^{k}$, and the target level $f_{\mathrm{lev}}^{k}<f\left(x^{k}\right)$ is chosen to ensure $f_{\mathrm{lev}}^{k} \rightarrow f^{*}:=\inf _{S} f$ as $k \rightarrow \infty$. If a finite lower bound $f_{\text {low }}^{k} \leq f^{*}$ is known, then usually $f_{\text {lev }}^{k}=f\left(x^{k}\right)-\kappa_{l} \Delta^{k}$, where $0<\kappa_{l}<1$ and the optimality gap $\Delta^{k}=f\left(x^{k}\right)-f_{\text {low }}^{k}$ provides the optimality estimate $f\left(x^{k}\right)-f^{*} \leq \Delta^{k}$. Since $\check{f}^{k} \leq f$, if the feasible set $S^{k}=\left\{x \in S: \check{f}^{k}(x) \leq f_{\text {lev }}^{k}\right\}$ of (1) is empty then $f_{\mathrm{lev}}^{k}<f^{*}$, so setting $f_{\mathrm{low}}^{k}=f_{\mathrm{lev}}^{k}$ reduces $\Delta^{k}$ by at least $\kappa_{l}$. Also $f_{\mathrm{lev}}^{k}$ is increased and $y^{k+1}$ is recomputed if the direction $d^{k}=y^{k+1}-x^{k}$ is 'too large' relative to the desired descent $\dot{\delta}^{k}=f\left(x^{k}\right)-f_{\text {lev }}^{k}$. A descent step to $x^{k+1}=y^{k+1}$ is taken if $f\left(y^{k+1}\right) \leq f\left(x^{k}\right)-\kappa_{d} \delta^{k}$, where $0<\kappa_{d}<1$ (i.e., if the actual descent is at least a fraction of the desired one). Otherwise, a null step $x^{k+1}=x^{k}$ provides a new linearization $f^{k+1}$.

The original level methods of Lemaréchal et al. (1995) require $S$ to be compact. They are hardly implementable because they employ $J^{k}=\{1: k\}$ and $f_{\text {low }}^{k}=\min _{S} \check{f}^{k}$. In contrast, our method needs bounded storage and is globally convergent without any compactness assumptions.

\section{THE DESCENT PROXIMAL LEVEL ALGORITHM}

The trial point finding subproblem (1) may be formulated as the QP problem

$$
\begin{array}{lll}
\text { minimize } & \left|x-x^{k}\right|^{2} / 2 & \text { over all } x \in S \\
\text { satisfying } & f^{j}(x) \leq f_{\text {lev }}^{k} & \text { for } j \in J^{k} .
\end{array}
$$

\section{Algorithm 1}

Step 0 (Initiation). Select an initial point $x^{1} \in S$, a final optimality tolerance $\epsilon_{\text {opt }} \geq 0$, a multiplier bound $t_{\max }>0$ and parameters $\kappa_{d}, \kappa_{l}, \kappa_{\delta} \in(0,1)$. Choose $f_{\text {low }}^{1} \leq f^{*}$ (e.g. , $\left.f_{\text {low }}^{1}=-\infty\right)$. Set $\Delta^{1}=f\left(x^{1}\right)-f_{\text {low }}^{1}$. Set $\delta^{1}=\kappa_{l} \Delta^{1}$ if $\Delta^{1}<\infty$; otherwise choose $\delta^{1}>0$. Set $J^{1}=\{1\}$. Set the counters $k=1, l=0$ and $k(0)=1$.

Step 1 (Level feasibility check). Set $f_{\mathrm{lev}}^{k}=f\left(x^{k}\right)-\delta^{k}$. If (2) is feasible, go to Step 3.

Step 2 (Update lower bound). Choose $f_{\mathrm{low}}^{k} \in\left[f_{\mathrm{lev}}^{k}, f^{*}\right]\left(\right.$ e.g.,$f_{\mathrm{low}}^{k}=f_{\mathrm{lev}}^{k}$ or $\left.\inf _{S} \check{f}^{k}\right)$. Set $\Delta^{k}=f\left(x^{k}\right)-f_{\text {low }}^{k}, \delta^{k}=\kappa_{l} \Delta^{k}$ and go to Step 1 .

Step 3 (Projection). Find the solution $y^{k+1}$ of (2) and its multipliers $\lambda_{j}^{k}$ such that the set $\hat{J}^{k}=\left\{j \in J^{k}: \lambda_{j}^{k}>0\right\}$ satisfies $\left|\hat{J}^{k}\right| \leq N$. Set $t^{k}=\sum_{j \in J^{k}} \lambda_{j}^{k}, d^{k}=y^{k+1}-x^{k}, p^{k}=-d^{k} / t^{k}$, $\tilde{f}_{S}^{k}=\check{f}^{k}\left(y^{k+1}\right)+\left\langle p^{k}, \cdot-y^{k+1}\right\rangle$ and $\tilde{\alpha}_{p}^{k}=f\left(x^{k}\right)-\tilde{f}_{S}^{k}\left(x^{k}\right)$.

Step 4 (Stopping criterion). If $\sigma^{k}:=\min \left(\Delta^{k}, \max \left\{\left|p^{k}\right|, \tilde{\alpha}_{p}^{k}\right\}\right) \leq \epsilon_{\mathrm{opt}}$, terminate.

Step 5 (Multiplier check). If $t^{k}>t_{\max }$, replace $\delta^{k}$ by $\kappa_{\delta} \delta^{k}$ and go to Step 1 .

Step 6 (Descent test). If $f\left(y^{k+1}\right) \leq f\left(x^{k}\right)-\kappa_{d} \delta^{k}$, set $t_{L}^{k}=1, k(l+1)=k+1$ and increase the counter of descent steps $l$ by 1 ; otherwise, set $t_{L}^{k}=0$ (null step). Set $x^{k+1}=x^{k}+t_{L}^{k} d^{k}$. 
Step 7 (Selection). Select $J_{s}^{k} \subset J^{k}$ such that $\hat{J}^{k} \subset J_{s}^{k}$. Set $J^{k+1}=J_{s}^{k} \cup\{k+1\}$.

Step 8 (Gap update). Set $f_{\text {low }}^{k+1}=f_{\text {low }}^{k}$ and $\Delta^{k+1}=f\left(x^{k+1}\right)-f_{\text {low }}^{k}$. If $t_{L}^{k}=0$, set $\delta^{k+1}=\delta^{k}$; otherwise, choose $\delta^{k+1} \in\left[\min \left\{\delta^{k}, \kappa_{l} \Delta^{k+1}\right\}, \Delta^{k+1}\right]$. Increase $k$ by 1 and go to Step 1 .

\section{MODIFIED LEVEL CONTROLS}

The level control of Algorithm 1 can be modified in order to increase its efficiency without destroying convergence. We list a few (implemented) possibilities below.

Suppose Step 1 finds another lower bound $\hat{f}_{\text {low }}^{k}$ of $f^{*}$ by computing $\inf _{S} \breve{f}^{k}$, or from a feasible point to the primal problem in Lagrangian relaxation. If $\hat{f}_{\text {low }}^{k} \geq f\left(x^{k}\right)-\hat{\kappa}_{l} \Delta^{k}$ for some fixed $\hat{\kappa}_{l} \in\left[\kappa_{l}, 1\right.$ ) (i.e., $\hat{f}_{\text {low }}^{k}$ is significantly better than $f_{\text {low }}^{k}$ ), then Step 2 may be entered to set $f_{\text {low }}^{k}=\hat{f}_{\text {low }}^{k}$ and reduce $\Delta^{k}$ by $\hat{\kappa}_{l}$.

If $\inf _{S} \check{f}^{k}$ is not computed, then $f_{\mathrm{lev}}^{k} \approx \inf _{S} \check{f}^{k}$ may be detected by the test $\left|p^{k}\right| \leq m_{\alpha} \tilde{\alpha}_{p}^{k}$ with a small $m_{\alpha}>0$. Hence Step 5 may use this additional test for decreasing $\delta^{k}$.

We may use the optimality measure $\tilde{\sigma}^{k}=\min _{j=1}^{k}\left\{\left|p^{k}\right|+\tilde{\alpha}_{p}^{k}\right\}$ for decreasing $\delta^{k}$ to $\min \left\{\kappa_{l} \Delta^{k}, \tilde{\sigma}^{k}\right\}$ and $\min \left\{\kappa_{\delta} \delta^{k}, \tilde{\sigma}^{k}\right\}$ at Steps 2 and 5 respectively, and for letting Step 8 choose $\delta^{k+1} \in\left[\min \left\{\delta^{k}, \kappa_{l} \Delta^{k+1}, \tilde{\sigma}^{k}\right\}, \Delta^{k+1}\right]$. This allows $\delta^{k}$ to decrease when $f\left(x^{k}\right)$ approaches $f^{*}$, as indicated by small $\left|p^{k}\right|$ and $\tilde{\alpha}_{p}^{k}$.

If the stepsize bound $t_{\max }$ is too small, the algorithm may crawl towards the solution. Hence our implementation sets $t_{\max }=100 t^{1}$, possibly increasing it to $\min \left\{10 t_{\max }, 10^{10}\right\}$ at Step 5 if $t^{k}>t_{\max }$ and more than two consecutive descent steps occured.

\section{SUBGRADIENT AGGREGATION}

To trade off storage and work per iteration for speed of convergence, one may replace subgradient selection with aggregation as in Kiwiel (1995b). Alternatively, one may employ selective aggregation Kiwiel (1995c) as follows. If we pick $\hat{\imath}, \hat{\jmath} \in J^{k}$ with $\lambda_{\hat{i}}^{k}, \lambda_{\hat{\jmath}}^{k}>0$, replace $f^{\hat{\jmath}}$ by $\left(\lambda_{\hat{\imath}}^{k} f^{\hat{\imath}}+\lambda_{\hat{j}}^{k} f^{\hat{\jmath}}\right) /\left(\lambda_{\hat{\imath}}^{k}+\lambda_{\hat{\jmath}}^{k}\right)$ and drop $\hat{\imath}$ from $J^{k}$, then the solution of (2) does not change.

\section{SOLVING QP AND LP SUBPROBLEMS}

Instead of using a separate LP solver for checking feasibility of (2) and possibly finding $\min _{S} \tilde{f}^{k}$, we employ the QP solver of Kiwiel (1989) within the exact penalty approach of Kiwiel (1995a). In this approach, given a penalty parameter $t>0$, one solves

minimize $\left|x-x^{k}\right|^{2} / 2+t \max \left\{\check{f}^{k}(x), f_{\mathrm{lev}}^{k}\right\} \quad$ over all $x \in S$.

Kiwiel (1995a) shows how to choose a sequence of penalty parameters so as to solve (2) or determine that (2) is infeasible and then deliver $\min _{S} f^{k}$. For handling large-scale problems, we intend to replace the QP solver of Kiwiel (1989) by that of Kiwiel (1994). 


\section{NUMERICAL EXPERIENCE}

We present comparisons of Algorithm 1 with our implementation of the simplest level method of Lemaréchal et al. (1995). In our notation it can be stated as follows:

\section{Algorithm 2}

Step 0 (Initiation). Select an initial point $x^{1} \in S$ and a final optimality tolerance $\epsilon_{\text {opt }} \geq 0$. If $S$ is unbounded, choose $f_{\text {low }}^{1} \in\left(-\infty, f^{*}\right]$. Set $J^{1}=\{1\}$.

Step 1 (Call oracle). Compute $f\left(x^{k}\right)$ and $g\left(x^{k}\right)$.

Step 2 (Compute lower bound). Compute $f_{\text {low }}^{k}=\inf _{S} \check{f}^{k}$.

Step 3 (Optimality test). Set $\Delta^{k}=f\left(x^{k}\right)-f_{\text {low }}^{k}$. If $\Delta^{k}<\epsilon_{\mathrm{opt}}$ then stop.

Step 4 (Projection). Set $f_{\text {lev }}^{k}=f_{\text {low }}^{k}+\Delta^{k} / 2$. Solve (2) to obtain $x^{k+1}$. Set $J^{k+1}=J^{k} \cup$ $\{k+1\}$. Increase $k$ by one and return to Step 1 .

Our implementations of Algorithms 1 and 2, called DPLM and LNN respectively, were programmed in MATLAB, using mex-interfaces to Fortran QP routines. LNN calls CPLEX for its LP subproblems. Our results for LNN deviate slightly from those of Lemaréchal et al. (1995), perhaps because a different LP solver was employed.

DPLM stores at most $N+10$ subgradients, whereas LNN stores all of them. For problems where $S$ is not compact we used a known lower bound on $f^{*}$ for both methods. We tested two versions of DPLM: one with $f_{\text {low }}^{k}=\max \left\{\inf _{S} \check{f}^{k}, f_{\text {low }}^{k-1}\right\}$ calculated by the QP solver only when (1) is infeasible, and one in which $f_{\text {low }}^{k}$ is calculated at every iteration using a separate LP solver. The latter version, which in the modified Step 1 used $\hat{\kappa}_{l}=0.9999$, is denoted by DPLM(LP). We used the parameters $\kappa_{d}=0.05, \kappa_{l}=0.8, \kappa_{\delta}=0.1$. The values of $f_{\text {low }}^{1}$ are specified for each example. Step 4 of DPLM used the stopping criterion $\Delta^{k} \leq \epsilon_{\text {opt }}$, unless stated otherwise.

\subsection{Standard test problems}

Table 1 gives results for standard test problems Kiwiel (1990), reporting iteration numbers at which the methods find solutions optimal to about six digits.

\subsection{Traveling salesman problems}

Table 2 gives results for Lagrangian minimum spanning tree relaxations of traveling salesman problems with $N$ cities, starting from the origin. Neither method solved the 442 node problem to the required accuracy; LNN had reached a value of -50434 after 600 iterations and DPLM had reached a value of -50499 after the same number of iterations.

\subsection{Eigenvalue problems}

Table 3 gives results for eigenvalue problems stated as $\min \lambda_{1}\left(M+P^{T} X Q+Q^{T} X^{T} P\right)$, where $M, P$ and $Q$ are constant matrices, $X$ is a matrix variable, and $\lambda_{1}(A)$ is the largest eigenvalue of a symmetric matrix $A$. In these five examples $X$ is $4 \times 5$ and $M$ is $7 \times 7$, and we used $f_{\text {low }}^{1}=-0.1, S=[-1,1]^{N}$ and $\epsilon_{\mathrm{opt}}=10^{-5}$. These problems are extremely 
Table 1 Standard test problems

\begin{tabular}{rrrrrrr}
\hline Problem & $N$ & $\epsilon_{\text {opt }}$ & $f_{\text {low }}^{1}$ & LNN & DPLM & DPLM(LP) \\
\hline MXQUAD & 10 & $10^{-5}$ & -10 & 74 & 60 & 60 \\
GOFFIN & 50 & $10^{-6}$ & -10 & 73 & 53 & 53 \\
HILB & 50 & $10^{-4}$ & -100 & 437 & 48 & 48 \\
TR48 & 48 & $10^{-0}$ & -700000 & 209 & 183 & 183 \\
SHUR & 5 & $10^{-4}$ & 0 & 40 & 33 & 36 \\
L1HILN & 10 & $10^{-6}$ & -10 & 32 & 22 & 22 \\
MINSUM & 6 & $10^{-4}$ & 0 & 54 & 50 & 44 \\
LOCATN & 4 & $10^{-5}$ & 0 & 24 & 31 & 32 \\
POLAK2 & 8 & $10^{-5}$ & -10 & 110 & 86 & 86 \\
\hline
\end{tabular}

Table 2 Travelling salesman problems

\begin{tabular}{rrrrrrr}
\hline$N$ & $f^{*}$ & $\epsilon_{\text {opt }}$ & $f_{\text {low }}^{1}$ & LNN & DPLM & DPLM(LP) \\
\hline 6 & -617.000 & $10^{-3}$ & -1000 & 15 & 32 & 33 \\
14 & -3322.000 & $10^{-2}$ & -4000 & 38 & 42 & 42 \\
29 & -2013.500 & $10^{-2}$ & -3000 & 90 & 78 & 77 \\
100 & -20937.950 & $10^{-1}$ & -30000 & 312 & 125 & 130 \\
120 & -6911.250 & $10^{-2}$ & -8000 & 434 & 211 & 210 \\
442 & -50505.675 & $10^{-1}$ & -51000 & & & \\
\hline
\end{tabular}

ill-conditionend. For termination we had to use the weaker criterion of Step 4 in DPLM and the corresponding one for LNN. DPLM(LP) performed exactly as DPLM.

Table 3 Eigenvalue problems

\begin{tabular}{rrrrr}
\hline & LNN & \multicolumn{2}{c}{ DPLM } \\
Problem & $f\left(x^{k}\right)$ & $k$ & $f\left(x^{k}\right)$ & $k$ \\
\hline EIG1 & $-2.03590866 \mathrm{e}-04$ & 234 & $-2.035181426 \mathrm{e}-04$ & 445 \\
EIG2 & $-1.14572767 \mathrm{e}-05$ & 204 & $-1.015554397 \mathrm{e}-05$ & 366 \\
EIG3 & $-3.89598493 \mathrm{e}-03$ & 296 & $-3.898228667 \mathrm{e}-03$ & 457 \\
EIG4 & $-2.80409331 \mathrm{e}-03$ & 326 & $-2.846003463 \mathrm{e}-03$ & 520 \\
EIG5 & $-1.51148612 \mathrm{e}-04$ & 223 & $-1.806084067 \mathrm{e}-04$ & 487 \\
\hline Mean & & 257 & & 455 \\
\hline
\end{tabular}


Table 4 Lotsizing problems

\begin{tabular}{|c|c|c|c|c|c|}
\hline \multicolumn{3}{|c|}{ Test problem } & \multirow[t]{2}{*}{ LNN } & \multirow[t]{2}{*}{ DPLM } & \multirow[t]{2}{*}{ DPLM(LP) } \\
\hline Cap. & Cost & $f^{*}$ & & & \\
\hline Tight & High & 27906 & 60 & 54 & 54 \\
\hline Tight & Med. & 7997 & 52 & 49 & 58 \\
\hline Tight & Low & 2893.3 & 20 & 15 & 15 \\
\hline Med. T & High & 24363 & 54 & 36 & 39 \\
\hline Med. T & Med. & 7722 & 27 & 28 & 35 \\
\hline Med. T & Low & 2893.3 & 13 & 15 & 19 \\
\hline Med. L & High & 20293 & 36 & 32 & 29 \\
\hline Med. L & Med. & 7534 & 21 & 15 & 19 \\
\hline Med. L & Low & 2865 & 1 & 1 & 1 \\
\hline Loose & High & 18872 & 20 & 14 & 21 \\
\hline Loose & Med. & 7464 & 13 & 13 & 12 \\
\hline \multirow[t]{2}{*}{ Loose } & Low & 2865 & 1 & 1 & 1 \\
\hline & & Mean & 26.5 & 22.75 & 25.25 \\
\hline
\end{tabular}

\subsection{Lotsizing problems}

The capacitated multi-item lotsizing problem is a scheduling model, which aims at scheduling production of several products over a planning horizon, while minimizing production costs, inventory holding costs and setup costs subject to demand and capacity constraints. The dual problem which is considered here arises from relaxing the capacity constraints using Lagragian multipliers. In each iteration we attempt to find a primal feasible solution in order to get a lower bound on the objective. The test problems have 8 variables which are constrained to the nonnegative orthant. For details, see Thizy and van Wassenhove (1985) and Brännlund (1993). We used $f_{\text {low }}^{1}=f^{*}$ and $\epsilon_{\mathrm{opt}}=10^{-6+\left[\log _{10}\left(\left|f^{*}\right|+1\right)\right\rceil}$, i.e. 6 digits of accuracy.

\subsection{Hierarchical Production Planning Problems}

A variation of the multi-item lotsizing problem was introduced by Graves (1982). The test problems, which are specified in the appendix II of Graves (1982), are randomly generated. They fall into 3 different test sets. The test problems have different level of capacity limits (Tight, Medium, and Loose) and different levels of setup costs (High, Medium, Low). Each test problem has 36 dual variables. We generate one random problem for each test set, each capacity limit and each setup cost. In each iteration we attempt to find a primal feasible solution in order to get a lower bound on the objective. For details, see Graves (1982) and Brännlund (1993). We used $f_{\text {low }}^{1}=f^{*}$ and $\epsilon_{\mathrm{opt}}=10^{-6+\left[\log _{10}\left(\left|f^{*}\right|+1\right)\right]}$, i.e. 6 digits of accuracy. 
Table 5 Hierarchical production planning problems

\begin{tabular}{|c|c|c|c|c|c|c|}
\hline \multicolumn{4}{|c|}{ Test problem } & \multirow[t]{2}{*}{ LNN } & \multirow[t]{2}{*}{ DPLM } & \multirow[t]{2}{*}{ DPLM(LP) } \\
\hline Set & Cap. & Cost & $f^{*}$ & & & \\
\hline 1 & Tight & High & 67435.0 & 143 & 130 & 143 \\
\hline 1 & Tight & Med. & 27560.3 & 129 & 184 & 152 \\
\hline 1 & Tight & Low & 13769.7 & 104 & 93 & 84 \\
\hline 1 & Med. & High & 60163.2 & 133 & 130 & 100 \\
\hline 1 & Med. & Med. & 21803.8 & 167 & 133 & 131 \\
\hline 1 & Med. & Low & 5666.1 & 101 & 68 & 68 \\
\hline 1 & Loose & High & 59118.5 & 121 & 112 & 124 \\
\hline 1 & Loose & Med. & 21792.0 & 99 & 64 & 63 \\
\hline 1 & Loose & Low & 5654.0 & 61 & 27 & 44 \\
\hline 2 & Tight & High & 67334.0 & 128 & 146 & 178 \\
\hline 2 & Tight & Med. & 28336.4 & 148 & 190 & 176 \\
\hline 2 & Tight & Low & 14541.6 & 94 & 77 & 79 \\
\hline 2 & Med. & High & 59698.9 & 138 & 116 & 150 \\
\hline 2 & Med. & Med. & 22015.2 & 149 & 180 & 132 \\
\hline 2 & Med. & Low & 7148.4 & 138 & 128 & 152 \\
\hline 2 & Loose & High & 58116.9 & 126 & 129 & 169 \\
\hline 2 & Loose & Med. & 21479.1 & 144 & 153 & 136 \\
\hline 2 & Loose & Low & 5597.8 & 69 & 56 & 55 \\
\hline 3 & Tight & High & 62346.1 & 88 & 110 & 129 \\
\hline 3 & Tight & Med. & 32719.1 & 133 & 143 & 154 \\
\hline 3 & Tight & Low & 22114.0 & 61 & 57 & 59 \\
\hline 3 & Med. & High & 55385.9 & 122 & 109 & 123 \\
\hline 3 & Med. & Med. & 26112.1 & 147 & 115 & 105 \\
\hline 3 & Med. & Low & 15911.3 & 79 & 66 & 83 \\
\hline 3 & Loose & High & 50000.5 & 102 & 99 & 98 \\
\hline 3 & Loose & Med. & 20712.6 & 142 & 125 & 124 \\
\hline \multirow[t]{2}{*}{3} & Loose & Low & 10408.3 & 77 & 103 & 70 \\
\hline & & & Mean & 116.4 & 112.7 & 114.1 \\
\hline
\end{tabular}

\subsection{Unit Commitment Problems}

The thermal unit commitment problem is a large mixed-integer non-linear mathematical programming problem which arises in short-term power production planning. The problem consists of minimizing production costs over a planning period, satisfying system load and reserve constraints as well as technical constraints on each production unit.

We refer to Wood and Wollenberg (1984) for a description of the unit commitment problem. Thorough descriptions of the BARD test problem can be found in Bard (1988), and for the EPRI50 in Zeminger et al. (1977). BARD2 is a relaxed modification of BARD. $\mathrm{ABB}$ and $\mathrm{ABB} 2$ can be obtained from the authors. We used $f_{\text {low }}^{1}=f^{*}$. 
Table 6 Unit commitment problems

\begin{tabular}{|c|c|c|c|c|c|c|}
\hline Test problem & $N$ & $f^{*}$ & $\epsilon_{\mathrm{opt}}$ & LNN & DPLM & DPLM(LP) \\
\hline BARD & 20 & 540952 & 1 & 399 & 709 & 386 \\
\hline BARD2 & 20 & 539923 & 1 & 334 & 751 & 260 \\
\hline $\mathrm{ABB}$ & 40 & 105973 & 1 & 97 & 77 & 84 \\
\hline $\mathrm{ABB} 2$ & 40 & 143696 & 1 & 138 & 72 & 93 \\
\hline \multirow[t]{2}{*}{ EPRI50 } & 96 & 2843720 & 10 & 309 & 156 & 330 \\
\hline & & & Mean & 255 & 353 & 231 \\
\hline
\end{tabular}

\section{REFERENCES}

Bard, J.F. (1988) Short-term scheduling of thermal-electric generators using Lagrangian relaxation. Operations Research, 36, 756-66.

Brännlund, U. (1993) On relaxation methods for nonsmooth convex optimization. $\mathrm{Ph} . \mathrm{D}$. thesis, Department of Mathematics, Royal Institute of Technology, Stockholm.

Brännlund, U., Kiwiel, K.C. and Lindberg, P.O. (1995) A descent proximal level bundle method for convex nondifferentiable optimization. Operations Research Letters (to appear).

Graves, S.C. (1982) Using Lagrangian techniques to solve hierarchical production planning problems. Management Science, 28, 260-75.

Kiwiel, K.C. (1989) A dual method for certain positive semidefinite quadratic programming problems. SIAM Journal on Scientific and Statistical Computing, 10, 175-86.

Kiwiel, K.C. (1990) Proximity control in bundle methods for convex nondifferentiable minimization. Mathematical Programming, 46, 105-22.

Kiwiel, K.C. (1994) A Cholesky dual method for proximal piecewise linear programming. Numerische Mathematik, 68, 325-40.

Kiwiel, K.C. (1995a) Finding normal solutions in piecewise linear programming. Applied Mathematics and Optimization, 32, (to appear).

Kiwiel, K.C. (1995b) Proximal level bundle methods for convex nondifferentiable optimization, saddle-point problems and variational inequalities. Mathematical Programming, (to appear).

Kiwiel, K.C. (1995c) The efficiency of subgradient projection methods for convex optimization, part II: Implementations and extensions. SIAM Journal on Control and Optimization, (to appear).

Lemaréchal, C., Nemirovskii, A.S. and Nesterov, Yu.E. (1995) New variants of bundle methods. Mathematical Programming, (to appear).

Thizy, J.M. and van Wassenhove, L.N. (1985) Lagrangean relaxation for the multi-item capacitated lot-sizing problem: A heuristic implementation. IIE Transactions, 17, 30813.

Wood, A.J. and Wollenberg, B.F. (1984) Power Generation Operation and Control. John Wiley and Sons, New York.

Zeminger, H.W., Wood, A.J., Clark, H.K., Laskowski, T.F. and Burns, J.D. (1977) Synthetic electric utility systems for evaluating advanced technologies. EM-285. Electrical Power Research Institute (EPRI). 